\title{
Efectos de la intervención educativa de enfermería en las prácticas de cuidados paliativos en el cuidador primario al paciente en enfermedad terminal. Lima, Perú.
}

\author{
De la Cruz Ruiz Daniel ${ }^{1, a}$, Torres Deza Clara ${ }^{2, b}$
}

\begin{abstract}
RESUMEN
Objetivos: determinar el efecto de la intervención educativa de enfermería en las prácticas de Cuidados Paliativos en el cuidador primario al paciente con enfermedad terminal. Material y métodos: diseño de estudio cuasi - experimental, la muestra estuvo constituida por 138 cuidadores primarios, se aplicó una guía de observación de 20 ítems en el pre test y post test, la validez fue medida a través del Análisis Factorial Exploratorio, aplicando el método muestral de Kaiser-Meyer-Olkin y la confiabilidad se evaluó según estadístico Kudder Richardson para variables dicotómicas en una muestra piloto (30 cuidadores primarios). Análisis de los datos fueron tabulados por el paquete estadístico SPSS v 20; y analizados con un nivel de significancia $<0,05$. Resultados: en cuanto a las prácticas de cuidados paliativos antes y después de la intervención educativa en el cuidador primario del paciente en enfermedad terminal según la guía de observación en el pre test, un 80,4\% presenta practicas inadecuadas, en el post test un $68,1 \%$ medianamente adecuada. En cuanto al puntaje de prácticas, antes de la intervención según guía de observación fue 6,86 puntos, mientras que después de la intervención se incrementó a 7,58 puntos, siendo la diferencia estadísticamente significativa $(\mathrm{p}=0.000)$. Conclusiones: la intervención educativa incrementa las prácticas en los cuidadores primarios, lo cual favorece los cuidados paliativos para brindar calidad de vida en los pacientes con enfermedad terminal.
\end{abstract}

PALABRAS CLAVES: Intervención educativa, práctica de cuidados paliativos, cuidadores, paciente con enfermedad terminal.

\section{Effects of the educational intervention of infirmary in the practices of taken care palliative in the primary keeper to the patient in terminal illness. Lima, Perú.}

\section{SUMMARY}

Objectives: to determine the effect of educational nursing intervention in palliative care practices in the primary caregiver to the patient with terminal illness. Material and methods: Study design quasi - experimental sample consisted of 138 primary caregivers, an observation guide of 20 items was applied in the pre test and post test, the validity was measured by exploratory factor analysis, applying the sampling method Kaiser -Meyer-Olkin and reliability was evaluated according to statistical Kudder Richardson for dichotomous variables in a pilot sample (30 primary caregivers). Analysis of the data was tabulated by SPSS v 20; and analyzed with a significance level $<0.05$. Results: in terms of palliative care practices before and after the educational intervention in the primary caregiver of the patient in terminal disease as the observation guide in the pretest, $80.4 \%$ have inadequate practices in the post test $68.1 \%$ suitable medium. As the score range, before the intervention by observation guide was 6.86 points, while after the intervention was increased to 7.58 points, with statistically significant difference $(\mathrm{p}=0.000)$. Conclusions: educational intervention increases practices primary caregivers, which promotes palliative care to provide quality of life in patients with terminal illness.

KEYWORDS: Educational Intervention, practice of Palliative Care, Caregiver, Patient with terminal illness.

\footnotetext{
Servicio de Medicina, Hospital Nacional Arzobispo Loayza. Lima, Perú.

Docente de la Facultad de Enfermería, Universidad Peruana Cayetano Heredia. Lima, Perú.

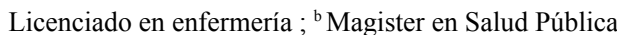




\section{INTRODUCCIÓN}

Los cuidados paliativos son tan antiguos como la medicina; sin embargo los estudios empíricos, recién comienzan hace cerca de 40 años en Gran Bretaña, la Dra. Cicely Saunders fue quien demostró que era posible morir en paz y sin grandes dolores, por ello fundó el primer hospicio St Christopher's, Sydenham en 1967; para la atención a pacientes terminales. La OMS define a los cuidados paliativos "como el cuidado total activo de los pacientes cuya enfermedad no responde a tratamiento curativo. El control del dolor y de otros síntomas y de problemas psicológicos, sociales y espirituales es primordial" (1).

En el Perú, en los últimos años, según el perfil epidemiológico las enfermedades crónicas degenerativas se han incrementado, tales como: enfermedad cardiaca, cáncer, enfermedades respiratorias y diabetes, que son las principales causas de mortalidad en el mundo, siendo estos responsables del 63\% de las muertes. En el año 2008, 36 millones de personas murieron de una enfermedad crónica, de las cuales la mitad era de sexo femenino y $29 \%$ era de menos de 60 años de edad(2).

La enfermedad terminal es aquella que se manifiesta como un padecimiento avanzado, progresivo e incurable, sin respuesta al tratamiento específico, con síntomas multifactoriales y pronóstico de vida no mayor a seis meses. Al no existir posibilidades de curación, los cuidados paliativos son la alternativa más viable en este contexto, para brindar calidad de vida y una muerte con dignidad y respeto. La tendencia actual en la atención al paciente con enfermedad terminal comprende la atención en el domicilio, asumiendo la atención un familiar que asume el rol de cuidador primario (3).

El cuidador primario o el familiar tienen una relación o vínculo directo con el paciente, que se hace cargo de proveer atención y asistencia y que generalmente vive momentos de incertidumbre. El cuidador primario generalmente no ha tenido formación y no encuentra la explicación o el conocimiento para afrontar las complicaciones propias y satisfacer las necesidades básicas para lograr una calidad de vida. El rol educador de Enfermería es generar cambios y brindar una herramienta segura al cuidador primario y evitar complicaciones en la salud del paciente con enfermedad terminal mejorando así la calidad de vida para tener una muerte tranquila $(4,5)$

El objetivo del presente trabajo fue determinar el efecto de la intervención educativa de enfermería dirigida a los cuidadores primarios en las prácticas de cuidados paliativos en el paciente con enfermedad terminal en el Hospital Nacional Arzobispo Loayza, Lima - Peru 2013.

MATERIAL Y MÉTODOS
El estudio es de diseño cuasi-experimental, la muestra estuvo constituida por 138 cuidadores primarios, se aplicó una guía de observación de 20 ítems en el pre test y post test, la validez fue medida a través del Análisis Factorial Exploratorio, aplicando el método muestral de Kaiser-Meyer-Olkin, y la confiabilidad se evaluó según estadístico Kudder Richardson para variables dicotómicas en una muestra piloto (30 cuidadores primarios). Análisis de los datos fueron tabulados por el paquete estadístico SPSS v 20; y analizados con un nivel de significancia $<0,05$.

\section{RESULTADOS}

El puntaje promedio de prácticas, antes de la intervención educativa según guía de observación fue 6.86 puntos, mientras que después de la intervención educativa se incrementó a 7.58 puntos, siendo la diferencia estadísticamente significativa. $(\mathrm{p}=0.000)$ (Tabla 1$)$.

En la tabla 2, se observa antes de la intervención educativa que la práctica de cuidados paliativos en el cuidador primario fue inadecuada en un $80,4 \%$ y medianamente adecuada en un $19,6 \%$; mientras que después de la intervención un $68,1 \%$ evidenció una práctica medianamente adecuada y un $2,2 \%$ adecuada.

En la tabla 3, se observa que en la práctica de cuidados paliativos en el cuidador primario en la dimensión apoyo emocional antes de la intervención fue inadecuado, en un $82 \%$ mientras que después de la intervención fue medianamente adecuado en un $31 \%$; respecto a la dimensión espiritual fue inadecuado en un $60 \%$ antes de la intervención y después de la intervención fue medianamente adecuado en un $40 \%$; en la dimensión dolor antes de la intervención fue inadecuado en un $17 \%$ y un $59 \%$ después de la intervención fue medianamente adecuado; en la dimensión alimentación fue de $61.6 \%$ mientras que después de la intervención fue medianamente adecuado $70 \%$; en cuanto a la dimensión de higiene se observa un $75 \%$ inadecuado antes de la intervención, asimismo después de la intervención fue medianamente adecuado en un $88 \%$; en la dimensión higiene antes de la intervención fue de un $75 \%$ inadecuada y un $63 \%$ medianamente adecuada después de la intervención, y en la dimensión confort y seguridad antes de la intervención fue de un $83 \%$ inadecuado y un $56 \%$ después de la intervención fue adecuada.

Tabla 1. Efecto de la Intervención Educativa dirigida a Cuidadores Primarios en la Práctica de Cuidados Paliativos en el Hospital Nacional Arzobispo Loayza 2013

\begin{tabular}{llllll}
$\begin{array}{l}\text { Prácticas de } \\
\text { cuidados Paliativos }\end{array}$ & $\mathrm{N}$ & $\bar{X}$ & DS & $\mathrm{Z}$ & $\mathrm{P}$ \\
\hline
\end{tabular}

$\begin{array}{llllll}\text { Antes } & 138 & 6,86 & 2,030 & & \\ \text { Después } & 138 & 7,58 & 2,669 & & \end{array}$


Tabla 2. Prácticas de Cuidados Paliativos antes y después de la Intervención Educativa en el Cuidador Primario del Paciente en Enfermedad Terminal en el Hospital Nacional Arzobispo Loayza 2013.

\begin{tabular}{|c|c|c|c|c|c|c|c|c|c|c|c|c|}
\hline \multirow[t]{2}{*}{ 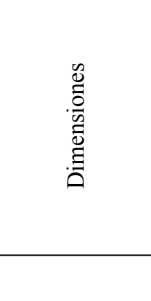 } & \multicolumn{2}{|c|}{$\begin{array}{l}\text { Ade- } \\
\text { cuada }\end{array}$} & \multicolumn{2}{|c|}{$\begin{array}{l}\text { Media- } \\
\text { namente } \\
\text { Adecuada }\end{array}$} & \multicolumn{2}{|c|}{$\begin{array}{l}\text { Inade- } \\
\text { cuada }\end{array}$} & \multicolumn{2}{|c|}{$\begin{array}{l}\text { Ade- } \\
\text { cuada }\end{array}$} & \multicolumn{2}{|c|}{$\begin{array}{l}\text { Me- } \\
\text { diana- } \\
\text { mente } \\
\text { Ade- } \\
\text { cuada }\end{array}$} & \multicolumn{2}{|c|}{$\begin{array}{l}\text { Inade- } \\
\text { cuada }\end{array}$} \\
\hline & $\mathrm{N}^{\mathrm{o}}$ & $\%$ & $\mathrm{~N}^{o}$ & $\%$ & $\mathrm{~N}^{0}$ & $\%$ & $\mathrm{~N}^{\mathrm{o}}$ & $\%$ & $\mathrm{~N}^{\circ}$ & $\%$ & $\mathrm{~N}^{\circ}$ & $\%$ \\
\hline $\begin{array}{l}\text { Apoyo } \\
\text { Emocional }\end{array}$ & - & - & 25 & 18 & 113 & 82 & - & - & 98 & 31 & 40 & 69 \\
\hline Espiritualidad & - & - & 34 & 25 & 104 & 75 & - & - & 55 & 40 & 83 & 60 \\
\hline Dolor & - & - & 115 & 83 & 23 & 17 & - & - & 82 & 59 & 56 & 41 \\
\hline Alimentación & - & - & 53 & 38.4 & 85 & 61.6 & - & - & 96 & 70 & 42 & 30 \\
\hline Eliminación & - & - & 46 & 33 & 92 & 67 & - & - & 95 & 88 & 43 & 12 \\
\hline Higiene & - & - & 35 & 25 & 103 & 75 & - & - & 87 & 63 & 51 & 37 \\
\hline $\begin{array}{l}\text { Confort y } \\
\text { Seguridad }\end{array}$ & - & - & 23 & 17 & 115 & 83 & 77 & 56 & 40 & 29 & 21 & 15 \\
\hline
\end{tabular}

Tabla 3. Nivel de las Prácticas de Cuidados Paliativos en el Cuidador Primario en el paciente con enfermedad terminal antes y después de recibir intervención Educativa según dimensiones en el Hospital Nacional Arzobispo Loayza 2013.

\begin{tabular}{lcccc}
\hline Nivel & $\begin{array}{c}\text { Antes de la interven- } \\
\text { ción educativa } \\
\text { No }\end{array}$ & $\begin{array}{c}\text { Después de la interven- } \\
\text { ción educativa } \\
\text { No }\end{array}$ & \multicolumn{2}{c}{ No } \\
\hline $\begin{array}{l}\text { Adecuada } \\
\text { Medianamente }\end{array}$ & - & - & 3 & 2,2 \\
$\begin{array}{l}\text { Adecuado } \\
\text { Inadecuada }\end{array}$ & 27 & 19.6 & 94 & 68,1 \\
Total & 111 & 80,4 & 41 & 29,7 \\
\hline
\end{tabular}

\section{DISCUSIÓN}

En el país el incremento de las enfermedades crónicas degenerativas con enfermedad terminal ha originado el incremento de la demanda en los servicios hospitalarios, en especial en los servicios de medicina. Los profesionales de enfermería satisfacen las demandas terapéuticas y las necesidades básicas de los pacientes con enfermedad terminal, empleando para ellos prácticas de cuidados paliativos de una forma integral y holística. Es por ello que el rol del enfermero se debe orientar, al acompañamiento, al apoyo y a la asesoría del cuidador primario por las demandas, satisfaciendo las demandas básicas del paciente con enfermedad terminal.

En la presente investigación se encontró que los cuidadores primarios que participaron se caracterizan por tener entre 31 a 40 años de edad, un $68,8 \%$ son de sexo feme- nino, un $98,6 \%$, estado civil solteros, un $94,2 \%$ con grado de instrucción de nivel secundario en un $92 \%$, ocupación independiente en un $76,1 \%$.

Estos resultados estarían relacionados con el estudio de Vega donde los cuidadores primarios son de género femenino, respecto de sus edades, la información general muestra que el $57 \%$ de los participantes se encuentran en el rango de 36 a 59 años y que el $26 \%$ tiene 60 años o más. También aparecen diferencias considerables en el estado civil de los cuidadores, porque la mayoría son solteras (6). Las razones primordiales sobre por qué la mayoría de cuidadores primarios son de sexo femenino, es la concepción de que la mujer está mejor preparada que el hombre para el cuidado, ya que tiene más capacidad de abnegación. Respecto a la práctica de cuidados paliativos (Tabla 1) realizado por el cuidador primario, en el pre test se obtuvo que el $80,4 \%$ presentaron prácticas inadecuadas antes de la intervención. Estos resultados estarían relacionados a que el cuidado es asumido generalmente por un miembro de la familia, que no tiene el conocimiento y prácticas sobre cuidados paliativos.

Según Mishel, la incertidumbre frente a la enfermedad es la incapacidad para determinar el significado de los hechos que guardan relación con la enfermedad, y aparecen cuando la persona que toma la decisión no es capaz de otorgar valores definidos a los objetos o hechos, o no es capaz predecir con decisión qué resultados se obtendrán. Cabe precisar si las situaciones originan estrés en las personas cuando pasan por momentos o situaciones inesperadas como el temor, miedo es por ello necesario la presencia de los profesionales para proporcionar asesoramiento permanente y continuo y disminuir o manejar las situaciones de incertidumbre que se dan en la cotidianidad (7).

Resultados similares fueron reportados por Valdés en el estudio "Conocimiento de familiares para el cuidado del enfermo terminal, donde obtuvo como resultado prácticas inadecuadas antes de la intervención educativa (8).

Por ello Henderson, refiere que las complicaciones propias de las atenciones se pueden evitar si los cuidadores primarios tuvieran el conocimiento, habilidad y destreza en el manejo de la atención en su paciente.

Asimismo en el post test se obtuvo que las prácticas inadecuadas disminuyó significativamente a un $29,7 \%$ y la práctica adecuada a un $2,2 \%$.

Estos resultados estarían relacionados con la eficacia de la intervención educativa debido a que se utilizó metodología participativa, basado en las necesidades básicas. Henderson considera que es necesario que el enfermero proporcione al paciente y familia los tres conceptos básicos: voluntad, fuerza y conocimiento para mantener la satisfacción de las 14 necesidades básicas con independen- 
cia, por ello el cuidador primario al brindar atención debe cumplir roles de apoyo en el hogar, que debe estar respaldada por la asesoría permanente por parte del profesional de enfermería, porque cuidar requiere voluntad, fuerza y conocimiento para mantener la calidad de vida (21).

El rol de la enfermera es el apoyo educativo, lo cual sustenta la importancia de la educación para que el cuidador primario asuma con responsabilidad y conocimiento la atención y conocimiento. Mishel encontró que ante la incertidumbre es necesaria la información al paciente en el cuidador primario, para la disminución del estrés y afrontamiento $(6,7)$.

Estudios similares como el de Vega, reportó un incremento significativo en las habilidades de cuidados familiares argumentando que el proceso de aprender a vivir con personas que presentan enfermedades crónicas, requiere un componente importante del conocimiento que le brinda un valor agregado a sus acciones y destacan en el conocimiento y la seguridad para la atención (6).

Al respecto, Tejeda efectuó una intervención educativa dirigida a los cuidadores familiares con enfermedad terminal. El conocimiento y las prácticas de los cuidadores familiares fue inadecuado en 18 de ellos, para 90,0\%, antes de la intervención educativa, después de realizada se observó cambios favorables en el grado de instrucción de 17 participantes, en un $85,0 \%$ (9).

Estos resultados podrían relacionarse con que la intervención educativa. Pues con el aprendizaje se evidencia cambios de comportamiento, actitud, pensamiento en el cuidador primario para resolver las necesidades originadas por la vulnerabilidad de la fragilidad del estado de salud del paciente con enfermedad terminal, se debe preparar al cuidador primario para resolver situaciones en la insatisfacción de las necesidades básicas de los pacientes; el enfermero utiliza en esta actividad educativa el conocimiento científico y las estrategias de su experiencia profesional para educar, sensibilizar y promover seguridad en el cuidador primario sobre la práctica de los cuidados paliativos en los pacientes con enfermedad terminal $(6,8)$.

Boff L, señala que el cuidado se caracteriza por elementos esenciales para la atención del paciente con enfermedad terminal dado que el conocimiento permite asumir este rol para satisfacer necesidades básicas y al desarrollo de la espiritualidad (10).

El puntaje promedio de prácticas, antes de la intervención educativa según guía de observación fue 6.86 puntos, mientras que después de la intervención educativa se incrementó a 7.58 puntos, siendo la diferencia estadísticamente significativa. $(p=0,000)$.

Al respecto Pender N, refiere que la promoción de la salud tiene como meta generar conductas y comportamiento saludable para disminuir los efectos adversos de la enfermedad, además, la autora señala que es importante que la persona identifique su meta y capacidad para el cambio, y la enfermera profesional debe apoyar en este proceso de cambio (7).

En cuanto al nivel de las prácticas de cuidados paliativos en el cuidador primario en el paciente con enfermedad terminal antes y después de recibir intervención educativa según dimensiones (Tabla 3) el apoyo emocional antes de la intervención fue inadecuada $82 \%$ mientras que después de la intervención disminuyó en un 13\%; respecto a la dimensión espiritual fue inadecuada en un $75 \%$ y después de la intervención disminuyó en un 15\%; en la dimensión dolor antes de la intervención fue inadecuada en un $66,6 \%$ mientras que después de la intervención educativa disminuyó $25 \%$, en cuanto a la dimensión alimentación fue inadecuada en un $61,6 \%$, y después de la intervención educativa disminuyó en un $29,4 \%$, en la dimensión eliminación fue inadecuada en un $67 \%$, después de la intervención educativa disminuyó en un $45 \%$, en la dimensión higiene fue inadecuada en un $75 \%$ después de la intervención educativa disminuyó en un $38 \%$ en cuanto a la dimensión confort y seguridad fue inadecuada en un $83 \%$ mientras que después de la intervención disminuyó en un $63 \%$.

Resultados similares obtuvo Vega en donde se mostró desconocimiento sobre todo en la técnica para realizar el baño en cama, el tratamiento que se debe tener con las sábanas y como aliviar en ellos las náuseas y vómitos. Por lo que concluyó que existe desconocimiento por parte de los familiares, de los cuidados a realizar a los pacientes en estado terminal (6).

Estos resultados podrían relacionarse con que la intervención educativa con el aprendizaje evidencia cambios de comportamiento, actitud, pensamiento en el cuidador primario para resolver las necesidades originadas por la vulnerabilidad de la fragilidad del estado de salud del paciente con enfermedad terminal, el enfermero utiliza en esta actividad educativa, el conocimiento científico y las estrategias de su experiencia profesional para educar, sensibilizar y promover seguridad en el cuidador primario sobre la práctica de los cuidados paliativos en los pacientes con enfermedad terminal $(6,8)$.

\section{CONCLUSIONES}

La práctica de los cuidadores primarios en la atención del paciente con enfermedad terminal muestran en el pre test que la mayoría presenta practicas inadecuadas, mientras que después de la intervención educativa la mayoría presentó prácticas medianamente adecuadas.

El efecto de la intervención educativa muestra un cambio 
estadísticamente significativo en el incremento de las prácticas de los cuidados paliativos en el paciente con enfermedad terminal.

En cuanto al nivel de las prácticas de cuidados paliativos en el cuidador primario en el paciente con enfermedad terminal antes y después de recibir intervención educativa según dimensiones, muestra un cambio significativo en el incremento del nivel de prácticas.

\section{RECOMENDACIONES}

Implementar programas de cuidado paliativo de enfermería en los servicios hospitalarios aplicando estrategia de motivación, control y seguimiento al cuidador primario. Realizar talleres prácticos sobre Cuidados Paliativos para el cuidador primario para satisfacer las demandas de las necesidades básicas en paciente en fase terminal.

Implementar una unidad de Cuidados Paliativos especializada dirigidos a los pacientes, familia y comunidad para garantizar una calidad de vida en los pacientes con enfermedad terminal en el Hospital Nacional Arzobispo Loayza.

\section{REFERENCIA BIBLIOGRÁFICAS}

1. Organización Mundial de la Salud. Cancer Pain Relief and Palliative Care. Technical Report Series 804., Ginebra: Organización Mundial de la Salud; 2005

2. Valderrama J. Acompañamiento del cuidador primario. 5ta ed. Madrid;2009.

3. Ruiz A, Ríos M. Cuidadores: responsabilidadesobligaciones. Mexico: ed. Medigrafic; 2012

4. Astudillo W. Atención Integral al paciente terminal (cuidado paliativos). ed. Madrid.

5. Rozo de A. Clara, Fundamentos pedagógicos que sustentan el proceso de la educación en salud. Aquichan. 2002; 2: 4.

6. Vega O, Mendoza M, Ureña M, Villamil W. Efecto de un programa educativo en la habilidad de cuidado de los cuidadores familiares de personas en situación crónica de enfermedad. Rev Ciencia y Cuidado, 2008; $1(5)$.

7. Raile A, Tomey M, Alligood M. Modelos y teorías en enfermería. 7ma Edición. Elsevier; 2011.

8. Valdés V. Conocimiento de familiares para el cuidado del enfermo terminal. Cuba: Municipio San José de las Lajas; 2007.

9. Tejeda D. La familia como agente terapéutico en los cuidados paliativos. Santiago de Cuba: Policlínico Universitario "José Martí Pérez" ;Diciembre 2009.

10.Boff L. Cuidado esencial. Scientific Circle; 2009. (Citado diciembre 2011) Disponible en: http://www. Scientificcircle.com/es/html.

\section{Correspondencia:}

Clara Torres-Deza

Correo electrónico: clara.torres@upch.pe

Fecha de Recepción: 21 de Octubre del 2015.

Fecha de aceptación: 07 de Diciembre del 2015. 